\title{
The Preventive Effect of Coffee Compounds on Dermatitis and Epidermal Pigmentation after Ultraviolet Irradiation in Mice
}

\author{
Yurika Yamate Keiichi Hiramoto Eisuke F. Sato \\ Department of Pharmaceutical Sciences, Suzuka University of Medical Science, Suzuka, Japan
}

\author{
Keywords \\ Ultraviolet · Coffee · Caffeic acid · Chlorogenic acid . \\ Dermatitis · Pigmentation
}

\begin{abstract}
Background: Ultraviolet (UV) irradiation is well known to promote inflammation and pigmentation of skin. UVB mainly affects dermatitis and pigmentation. Coffee contains a number of polyphenols, such as caffeic acid (CA) and chlorogenic acid (CGA) but their in vivo bioactivity for photobiology remains unclear. Methods: $\mathrm{C} 57 \mathrm{BL} / 6 \mathrm{j}$ male mice were irradiated with UVB $\left(1.0 \mathrm{~kJ} / \mathrm{m}^{2} /\right.$ day $)$ for 3 days. Five days after the final session of UVB irradiation, the dorsal skin, ear epidermis, and blood samples were analyzed to investigate the inflammatory factors, melanogenesis factors and related hormones. Results: After the oral administration of CA (100 $\mathrm{mg} /$ day) or CGA (100 mg/day) for 8 days, only CA was found to inhibit dermatitis and pigmentation. The pathway by which CA inhibits dermatitis is related to the mitogen-activated protein kinase (MAPK)/extracellular signal regulated kinase (ERK)1/2/CAMP response element binding protein (CREB) pathway. Otherwise, the pathway by which CA inhibits pigmentation is related to the activation of the $\beta$-endorphin- $\mu$-opioid receptor and suppresses the CAMPmicrophthalmia-associated transcription factor (MITF) path-
\end{abstract}

way. Conclusion: It is suggested that the oral administration of CA prevented dermatitis and pigmentation after UVB irradiation in mice.

(c) 2017 S. Karger AG, Basel

\section{Introduction}

The danger of ultraviolet (UV) irradiation has become a major societal problem, due to reports of a potential increase of $30-35 \%$ in UV irradiation on the surface of the earth in the coming years [1-3]. Caution must be exercised, as the increased irradiation will effect changes in humans, other animals, and plants. Although the UVB irradiation that reaches the surface represents only $4-10 \%$ of the total environmental UV light, it has a stronger phototoxic effect than UVA irradiation because DNA shows maximum absorption at approximately $260 \mathrm{~nm}$, with the absorption tail falling in the UVB region $[4,5]$. UVB is the most important modifiable risk factor for inflammation, pigmentation, skin cancer, and many other environmentally influenced skin disorders. The skin covers the surface of the body and protects against environmental stressors such as infectious pathogens, chemical agents, and UV radiation. Its protection is therefore vital.

\section{KARGER}

(c) 2017 S. Karger AG, Basel

E-Mail karger@karger.com

www.karger.com/spp
Yurika Yamate, $\mathrm{PhD}$

Department of Pharmaceutical Sciences

Suzuka University of Medical Science

3500-3 Minamitamagakicho, Suzuka, Mie 513-8670 (Japan)

E-Mail m8645767@ msic.med.osaka-cu.ac.jp 


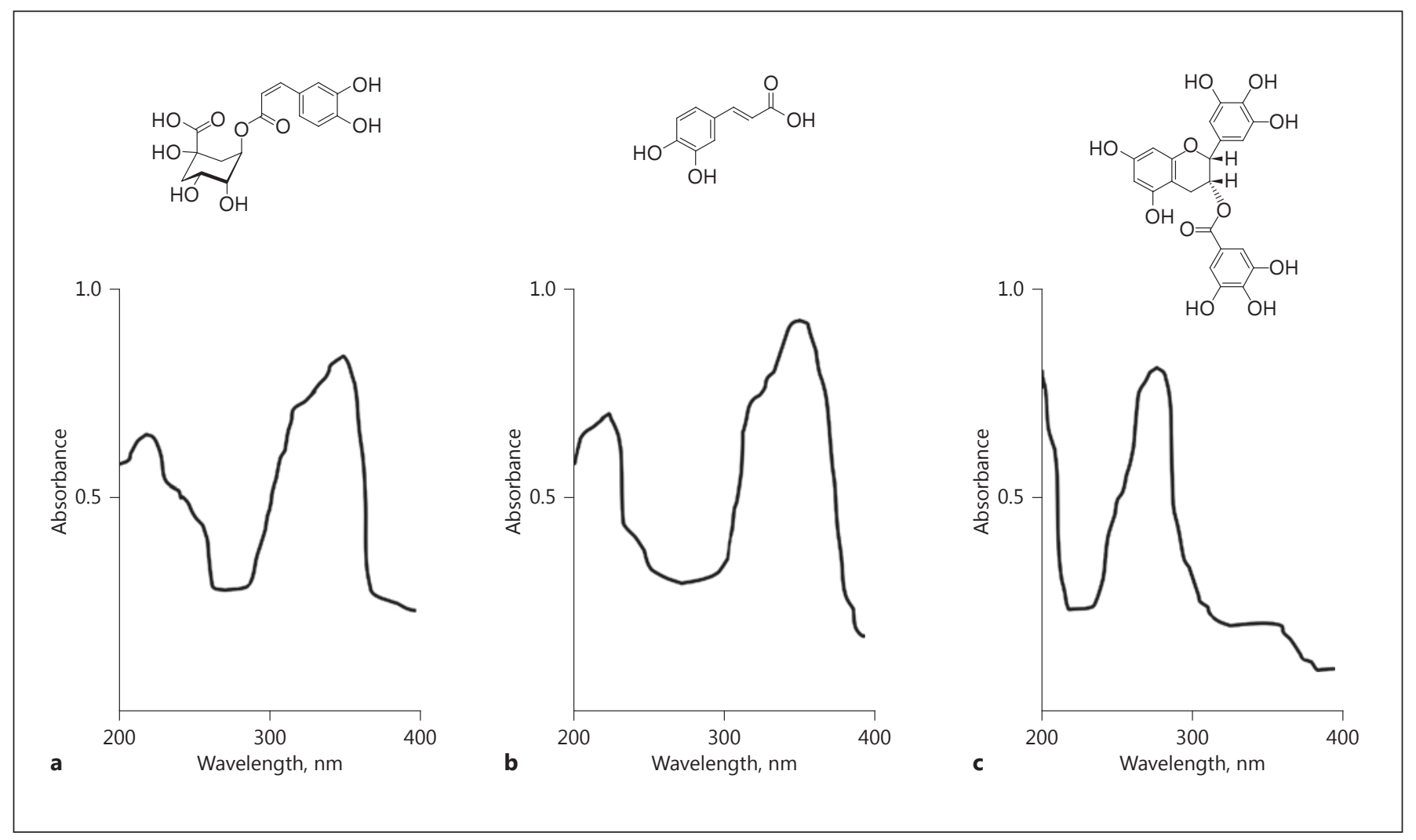

Fig. 1. The chemical structures and the UV absorbance spectra of CA, CGA, and EGCG. a CGA (5-o-caffeoylquinic acid). b CA (3,4-dihydroxycinnamic acid). c EGCG (epigallocatechin-3-gallate).

Coffee is a popular drink worldwide. In Japan, the number of people who drink coffee on a daily basis is increasing. Coffee contains a variety of chemical components [6]. In the present study, we focused on 2 components of coffee: caffeic acid (CA) and chlorogenic acid (CGA), both of which are well-known antioxidants $[7,8]$ (Fig. 1). While a number of reports have examined the anti-inflammation and antioxidative stress properties of these components [9-11], many of these studies were conducted as cellular experiments (in vitro), and few reports have been published on in vivo experiments.

We therefore investigated the protective effects of orally administered CA (100 mg/kg/day) or CGA (100 $\mathrm{mg} / \mathrm{kg} /$ day) against dermatitis and pigmentation after UVB irradiation in an in vivo study. Epigallocatechin3-gallate (EGCG), the most active compound in the catechin family of green tea extracts, has been shown to have both anti-inflammatory and antioxidant properties on multiple cell types in in vitro studies $[12,13]$. We therefore decided to use it as a comparative group against our CA and CGA groups.

Coffee and Ultraviolet
We herein reveal new effects of CA and CGA in the skin, due to their protective effects against dermatitis and pigmentation after UVB irradiation. If the chemical components of coffee are found to have protective biological effects, then these new beneficial effects of coffee consumption may receive greater focus.

\section{Materials and Methods}

\section{Animals}

All of the animals were treated in accordance with the animal care regulations of Suzuka University of Medical Science. Specific-pathogen-free, 8-week-old male C57BL/6j mice (SLC, Hamamatsu, Aichi, Japan) were used in the experiments. Under light sevoflurane anesthesia, the dorsal fur of the mice was shaved using electric clippers. Then, the whole body of the mouse was exposed to UVB irradiation (wave length range: $280-320 \mathrm{~nm}$, the wavelength of the light source peaked at $305 \mathrm{~nm}$ ) using a sunlamp (FL-20SE; Toshiba Co., Tokyo, Japan) for 3 days at a dose of 1.0 $\mathrm{kJ} / \mathrm{m}^{2}$ per day (irradiation time: $45 \mathrm{~s} /$ day), with the animals kept under light anesthesia. We filtered out other wavelengths using Kodaul cellulose film (Eastmann Kodak Co., Rochester, NY, 
USA). This dose was based on that used in our previous study and the typical 1-h daytime doses received by humans in Osaka, Japan [14].

\section{Chemicals}

CA was purchased from MP Biomedicals (Tokyo, Japan), CGA was purchased from Cayman Chemicals (Ann Arbor, MI, USA), and EGCG, a polyphenol found in green tea, was purchased from Tocris Bioscience (Bristol, UK). In the oral administration experiments, the concentrations of CA, CGA, and EGCG were all 100 $\mathrm{mg} / \mathrm{kg}$, as this is the concentration traditionally used in animal experiments such as the experiments performed in the previous study [15-17]. These chemical reagents were dissolved in $0.5 \%$ $(\mathrm{w} / \mathrm{v})$ carboxymethyl cellulose solvent and administered by oral gavage before UVB irradiation. The mice were divided into the following 6 groups: the control group (nontreatment), the UVB irradiation-only group, the UVB irradiation $+0.5 \%$ carboxymethyl cellulose solution group, and the UVB irradiation + CA, CGA, or EGCG groups. There were 6 animals in each group.

\section{The Draize Score}

For the analysis of dermatitis degrees 5 days after UVB irradiation, the site was examined for erythema and edema using a modified version of the Draize scoring system, with scores ranging from 0 to $8[18,19]$. The degree of erythema and edema was determined based on the scores for erythema: index value; $0=$ no erythema, 1 = very slight erythema (barely perceptible), 2 = well-defined erythema, $3=$ moderate to severe erythema, and $4=$ severe erythema; and edema formation: index value; $0=$ no edema, $1=$ very slight edema, 2 = slight edema, 3 = moderate edema, and $4=$ severe edema (extending beyond the area of exposure).

\section{The Preparation and Staining of the Dorsal Skin and}

Ear Epidermis

For the histological studies, the mice were sacrificed 5 days after the final session of UVB irradiation, and dorsal skin and ear epidermis samples were prepared. The dorsal skin was used for an inflammatory analysis, and the ear epidermis was used for a pigmentation analysis, because numerous melanocytes are commonly found in the ear epidermis of mice, while few are found in the dorsal skin. Thus, our examination of the ear epidermis of mice was in line with the methods of previous skin pigmentation studies involving mice [20]. To evaluate the inflammatory changes, the dorsal skin samples were observed via hematoxylin-eosin staining. For the analysis of pigmentation (melanogenesis), ear skin sheets $(0.5 \times 0.5 \mathrm{~cm})$ were prepared and incubated for $2 \mathrm{~h}$ at $37^{\circ} \mathrm{C}$ in $2 \mathrm{~mol} / \mathrm{L}$ sodium bromide. The epidermis was then separated from the dermis to obtain epidermal sheets. The staining for L-3,4-dihydroxyphenylalanine(DOPA)-positive melanocytes was performed via the method of Jimbow and Uesugi [20]. Briefly, after washing the epidermal sheets with phosphate-buffered saline (PBS), the sheets were incubated at $37^{\circ} \mathrm{C}$ in PBS containing $0.1 \%$ DOPA (Sigma, St. Louis, MO, USA). The sheets were then washed in PBS, fixed overnight at $25^{\circ} \mathrm{C}$ in $10 \%$ formalin, dehydrated, and stained for DOPA-positive melanocytes. All of the epidermal sheets were examined under coded conditions by 1 investigator. Approximately 20 fields of view (magnification: 20x) were chosen for each of the epidermal sheets, and a stack of approximately 30 optical sections was scanned with a $\mathrm{z}$-increment of $0-4 \mu \mathrm{m}$. From each optical stack, extended focus projections (summation of all optical sec- tions in the stack with each section in focus) were performed, and the areas of the cells were calculated using the software program provided with the BioRad MRC500 instrument (BioRad, Hercules, CA, USA). The number of DOPA-positive melanocytes per square millimeter was determined by counting 15-20 fields.

\section{A Western Blot Analysis of the Dorsal Skin and the Ear Skin}

In the oral administration experiments, the dorsal skin and the ear skin samples of the control group, UVB-only group, and UVB + CA group 1 day after UVB irradiation were separately homogenized in a lysis buffer containing $0.5 \%$ Nonidet P-40, $10 \%$ glycerol, $137 \mathrm{~mm} \mathrm{NaCl}, 2 \mathrm{~mm}$ ethylenediamine tetraacetic acid, and $50 \mathrm{~mm}$ Tris-HCl buffer ( $\mathrm{pH} 8.0$ ). After centrifugation at $8,000 \mathrm{~g}$ for $10 \mathrm{~min}$, the supernatant fractions were separated and stored at $-80^{\circ} \mathrm{C}$ until analysis. After thawing, equal amounts of the protein $(20.0 \mathrm{mg} /$ lane) were loaded onto a 4-12\% BIS-TRIS Blot gel (Life Technologies, Carlsbad, CA, USA) and electrophoresed at $200 \mathrm{~V}$ for $20 \mathrm{~min}$. Following separation, the proteins were transferred to a nitrocellulose membrane using an iBlot Western blotting system (Life Technologies), which was subsequently blocked with $5 \%$ skimmed milk at $4^{\circ} \mathrm{C}$ overnight. After blocking, the membranes were then incubated at $25^{\circ} \mathrm{C}$ for $1 \mathrm{~h}$ with primary antibodies against p-MAPK (phospho-mitogen-activated protein kinase; 1:1,000; Cell Signaling Technology Inc., Danvers, MA, USA), p-ERK (phospho-extracellular signal-regulated kinase; 1:1,000; Cell Signaling Technology Inc.), p-p38 (phospho-p38; 1:1,000; Cell Signaling Technology Inc.), $\mathrm{p}-\mathrm{AKT}$ (phospho-58-kDa serine/threonine protein kinase; 1 : 1,000; Cell Signaling Technology Inc.), p-CREB (phospho-cAMP response element binding protein; 1:1,000; Cell Signaling Technology Inc.), MC1R (melanocortin receptor-1; 1:1,000; Santa Cruz Biotechnology Inc., Santa Cruz, CA, USA), cAMP (cyclic adenosine monophosphate; 1:1,000; Santa Cruz Biotechnology Inc.), and $\beta$-actin (1:5,000; Sigma). Immunocomplexes on the membranes were then visualized using a horseradish peroxidase-conjugated secondary antibody (Dako Cytomation, Glostrup, Denmark) and immunoStar Zeta (Wako, Osaka, Japan). Images were acquired using the Multi-Gauge software program (Fujifilm, Tokyo, Japan).

The Quantification of $\alpha$-Melanocyte-Stimulating Hormone, Adrenocorticotropic Hormone and $\beta$-Endorphin in Plasma by an Enzyme-Linked Immunosorbent Assay

In the oral administration experiments, blood samples were taken from the heart 1 day after the final session of UVB irradiation. The plasma was then fractionated, and the plasma levels of $\alpha$-melanocyte-stimulating hormone ( $\alpha$-MSH), adrenocorticotropic hormone $(\mathrm{ACTH})$ and $\beta$-endorphin were determined using a commercial enzyme-linked immunosorbent assay (ELISA) kit (Phoenix Pharmaceuticals Inc., Hayward, CA, USA) in accordance with the manufacturer's instructions.

\section{Statistical Analysis}

All data are presented as the means \pm standard deviation (SD) of the values for 6 animals in each group. The statistical analyses were performed according to the SPSS Institute User's Guide. The analyses of variance (ANOVAs) were performed using the ANOVA procedure. Significant differences $(p<0.05)$ between the means were determined using the Tukey post hoc test. The SPSS software program (version 20) was used for the statistical analyses. $p$ values of $<0.05$ were considered to indicate statistical significance. 

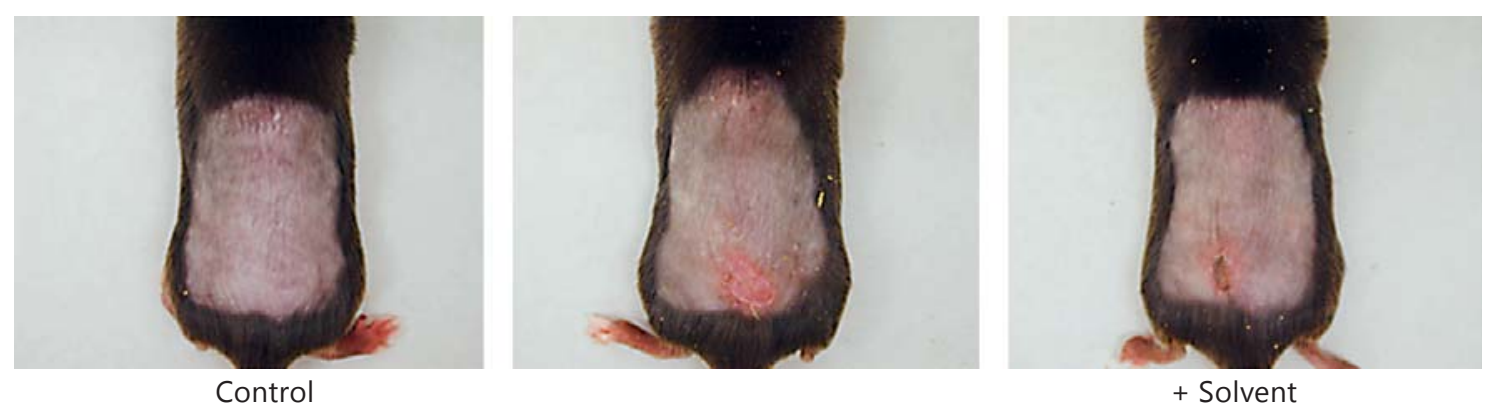

+ Solvent

UVB

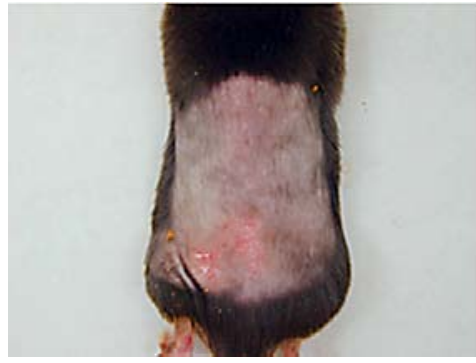

+ CGA

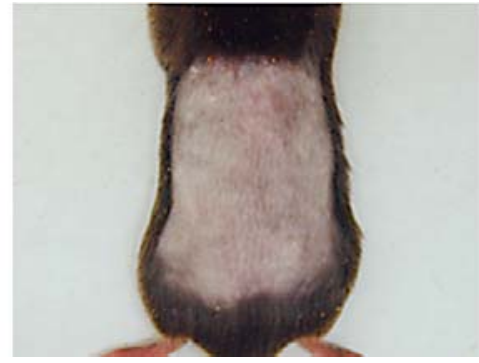

$+\mathrm{CA}$

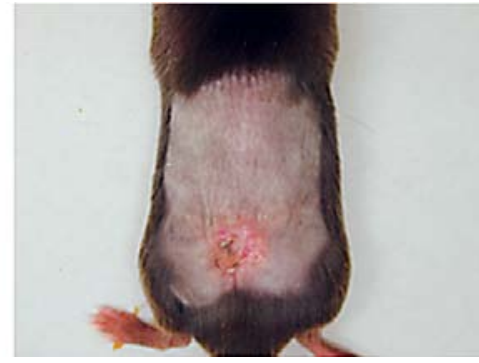

+ EGCG

a

UVB

Fig. 2. The effect of the coffee compounds on dorsal skin dermatitis 5 days after UVB irradiation. a The representative macroscopic images of dorsal skin in mice from each group 5 days after the final UVB irradiation session. b The Draize scores of the dorsal skin samples in mice 5 days after the final UVB irradiation session. The data were expressed as the mean \pm SD of 6 animals per group. A statistical analysis was performed using an ANOVA followed by the Tukey post hoc test using the SPSS (version 20) software program (biological replicates; ${ }^{*} p<0.05$ ).

\section{Results}

The Preventive Effect of CA against Skin

Inflammation after UVB Irradiation

In the oral administration experiments, the dorsal skin of mice showed marked dermatitis, inflammation, and

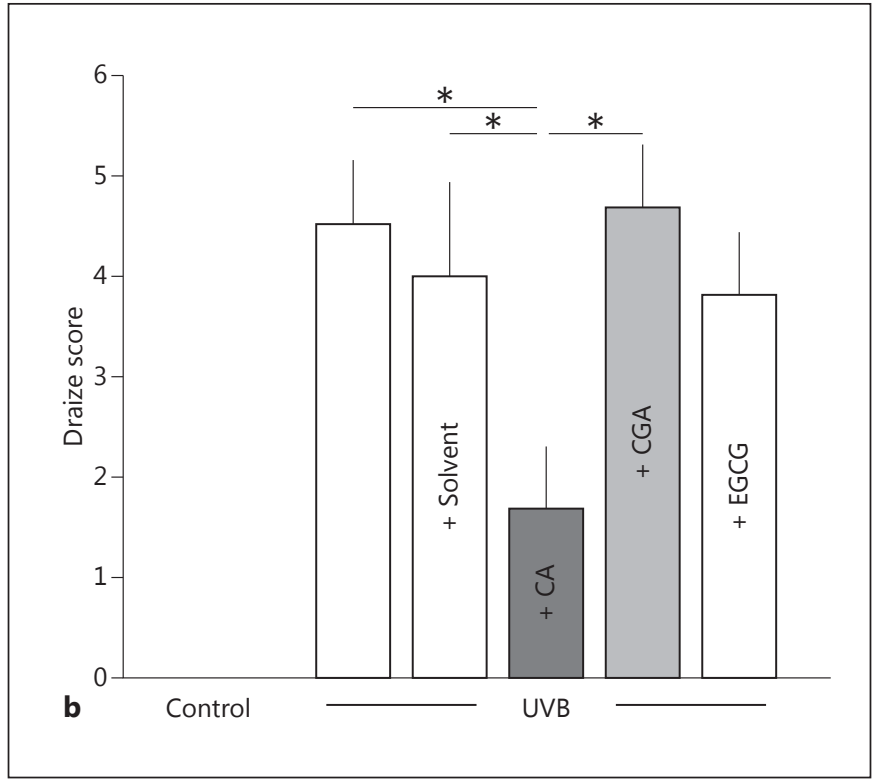

erythema 5 days after UVB irradiation (Fig. 2a). However, these skin inflammation symptoms were strongly inhibited in the UVB irradiation + CA group, although similar effects were not observed in the UVB irradiation + CGA group. Furthermore, the Draize score, which describes the degree of erythema and edema in skin, was 


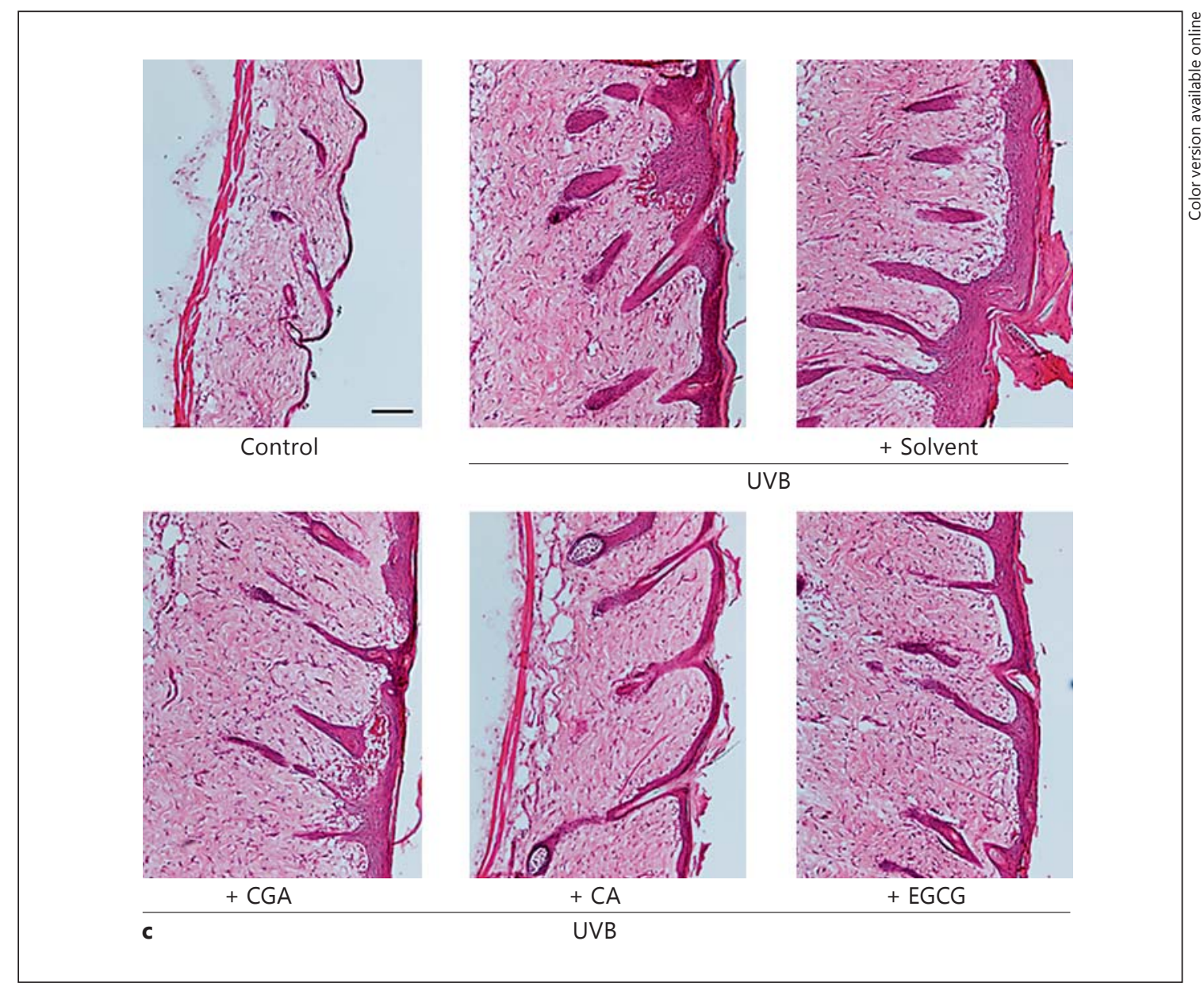

Fig. 2. The effect of the coffee compounds on dorsal skin dermatitis 5 days after UVB irradiation. c The histological analysis of the dorsal skin samples in mice 5 days after UVB irradiation using hematoxylin-eosin staining. The data reflect 1 typical experiment with 6 animals per group. Scale bar $=100 \mu \mathrm{m}$.

significantly decreased only in the UVB irradiation + CA group in comparison to the UVB irradiation group, the UVB + solvent group and the UVB + CGA group (Fig. 2b). The dorsal skin samples were stained for our histological analysis using hematoxylin-eosin (Fig. 2c). Five days after UVB irradiation, the dorsal skin epidermis in the UVB irradiation group was thicker than that in the control group, and many inflammatory cells were found to have invaded the dermis and edema of the dorsal skin. In contrast, these changes were markedly inhibited in the UVB irradiation + CA group. Of note, however, no such inhibition was noted in either of the groups receiving CGA or EGCG administration. These results indicate that the oral administration of CA was more effective for inhibiting skin inflammation than CGA or EGCG.
The Preventive Effect of CA against Skin Melanogenesis after UVB Irradiation

In the oral administration experiments, the ear epidermal sheets were prepared and stained using L-DOPA 5 days after UVB irradiation (Fig. 3a), and the number of DOPA-positive melanocytes was counted using a confocal laser scanning microscope (Fig. 3b). In the UVB irradiation or UVB + solvent groups, the numbers of DOPApositive melanocytes were markedly increased compared with the control group. This increase was subsequently inhibited in the UVB irradiation + CA group but not in the groups receiving CGA or EGCG administration. These results indicate that the oral administration of CA was more effective in inhibiting skin melanogenesis after UVB irradiation than CGA or EGCG. 


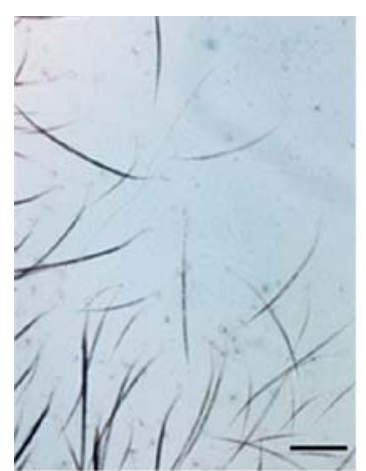

Control

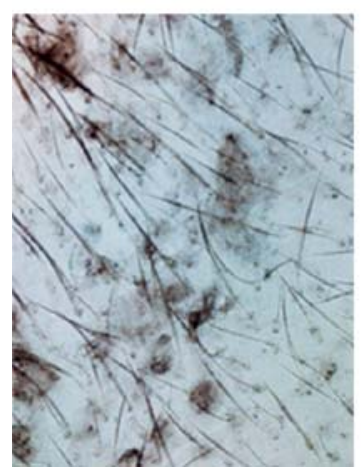

+ CGA
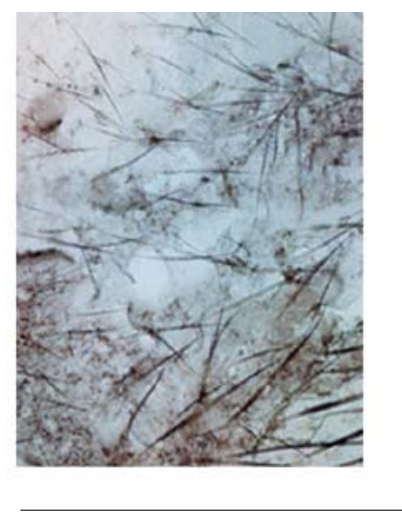

UVB

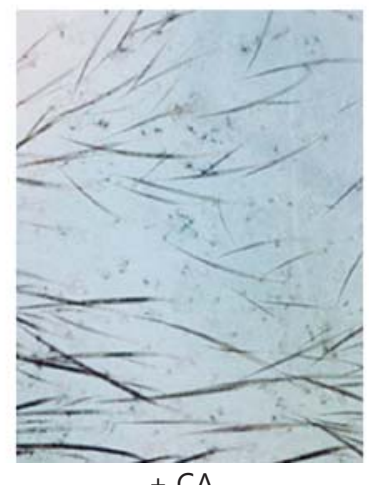

$+C A$

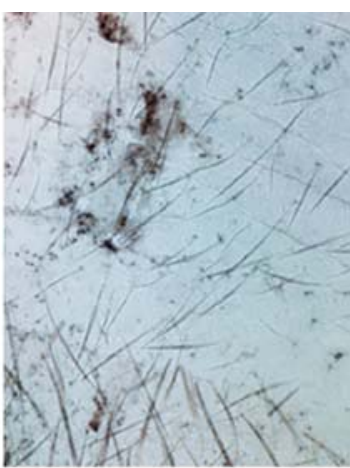

+ Solvent

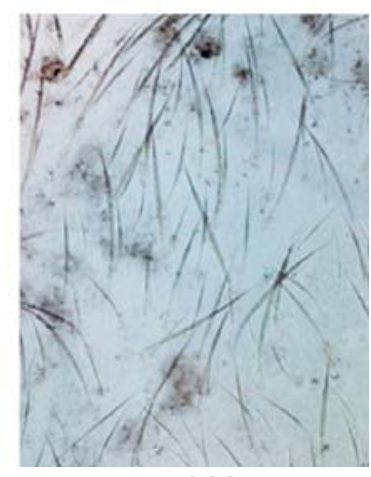

+ EGCG

a

Fig. 3. The effect of the coffee compounds on ear epidermal pigmentation 5 days after UVB irradiation. a The results of the histological analysis of the ear epidermal skin using DOPA staining. The numbers of DOPA-positive melanocytes were determined. The data reflect 1 typical experiment with 6 animals per group. Scale bar $=$ $100 \mu \mathrm{m}$. b The number of DOPA-positive melanocytes in the ear epidermal skin 5 days after UVB irradiation. The values were presented as the mean $\pm \mathrm{SD}$ derived from 6 animals per group. A statistical analysis was performed using an ANOVA followed by the Tukey post hoc test using the SPSS (version 20) software program (biological replicates; * $p<0.05$ ).

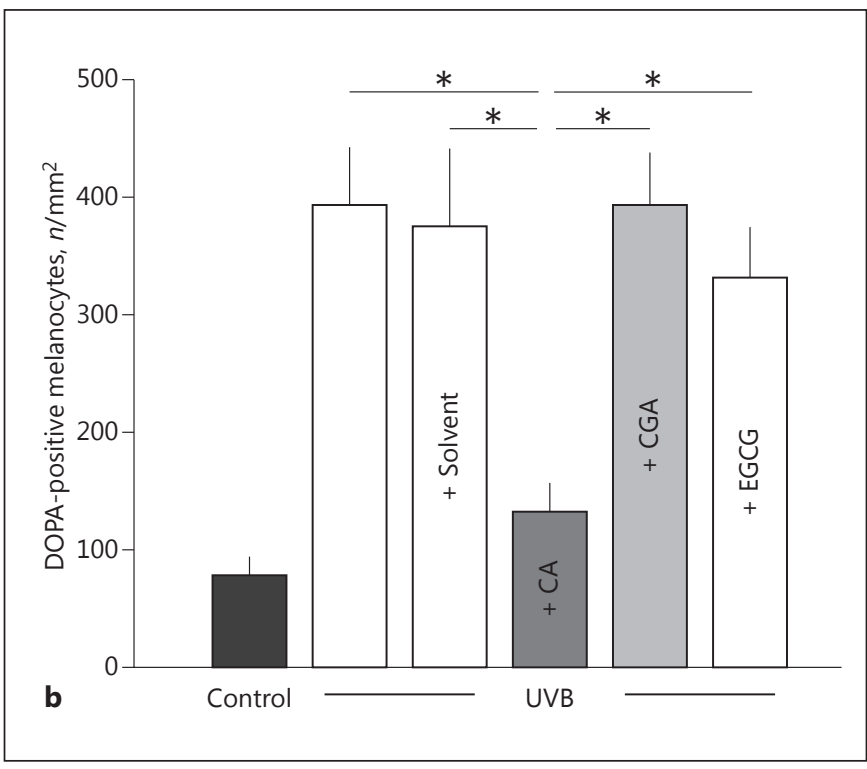

Skin Pharmacol Physiol 2017;30:24-35 


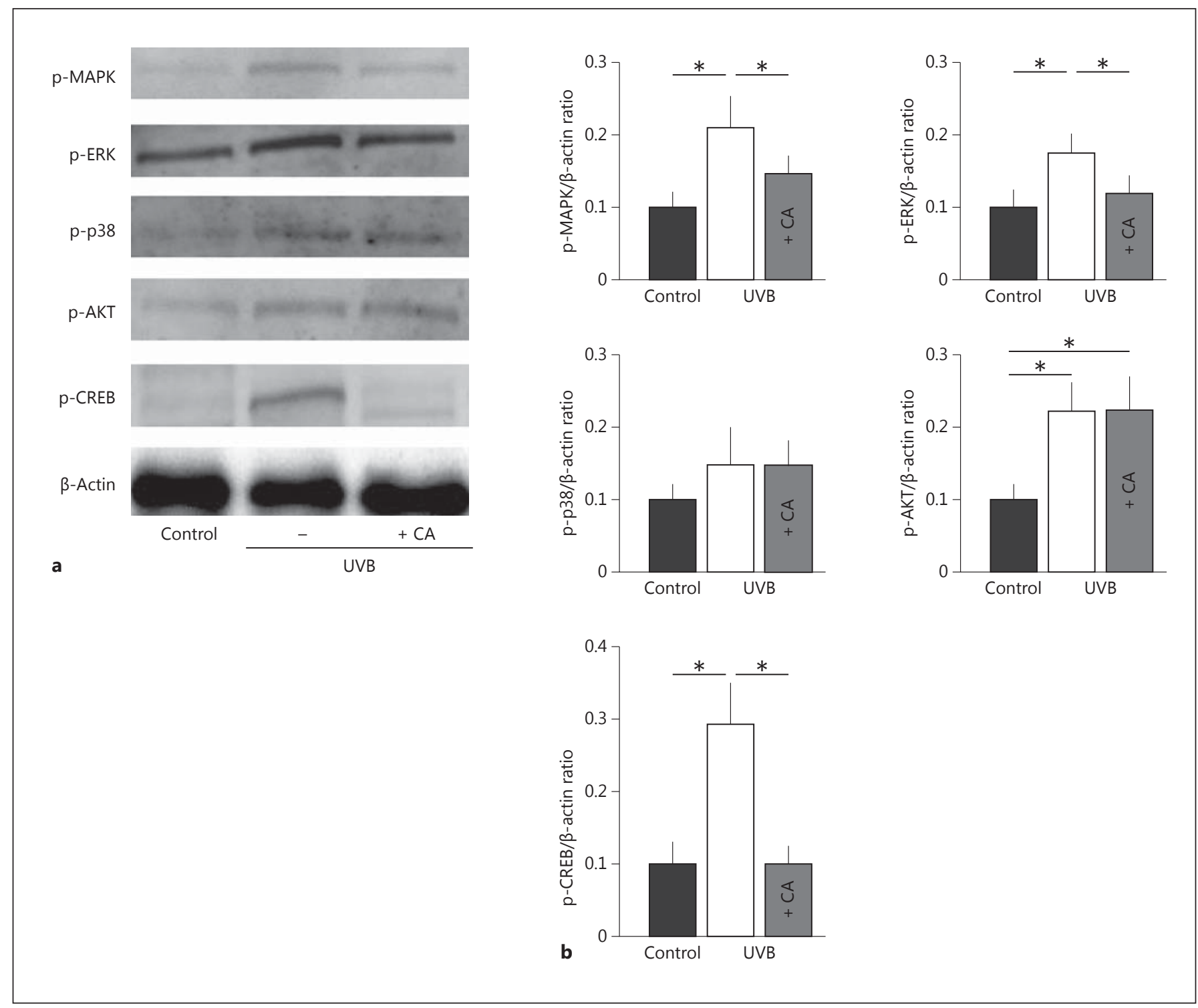

Fig. 4. The biochemical analysis of the mechanism underlying the dorsal skin inflammation 1 day after UVB irradiation. a The expression levels of p-MAPK, p-ERK1/2, p-p38, p-AKT, p-CREB, and $\beta$-actin were determined by Western blot analysis in the dorsal skin samples of each group. Equal protein loading was assessed using mouse $\beta$-actin. b Each band was quantified via densitometry.

The Mechanism Underlying the Preventive Effect of

$C A$ against Skin Inflammation after UVB

To investigate the mechanism underlying the preventive effect of CA against skin inflammation after UVB irradiation, we prepared dorsal skin samples 1 day after the final UVB irradiation session, when skin inflammation signals are commonly observed following UVB irradiation. We hypothesized that the skin inflammation might
The densitometry data were normalized for $\beta$-actin. The results are shown as the mean \pm SD derived from 6 animals per group. A statistical analysis was performed using an ANOVA followed by the Tukey post hoc test using the SPSS (version 20) software program (biological replicates; ${ }^{*} p<0.05$ ).

be controlled at the point of the signal system where CA was located and therefore analyzed the dorsal skin after oral administration of CA using Western blotting (Fig. 4a). The intensities of these data are shown in graphs of Figure $4 \mathrm{~b}$. Because we considered that the important groups for observation were the control group, UVB irradiation group, and UVB + CA group, we therefore analyzed only these 3 groups' samples. 


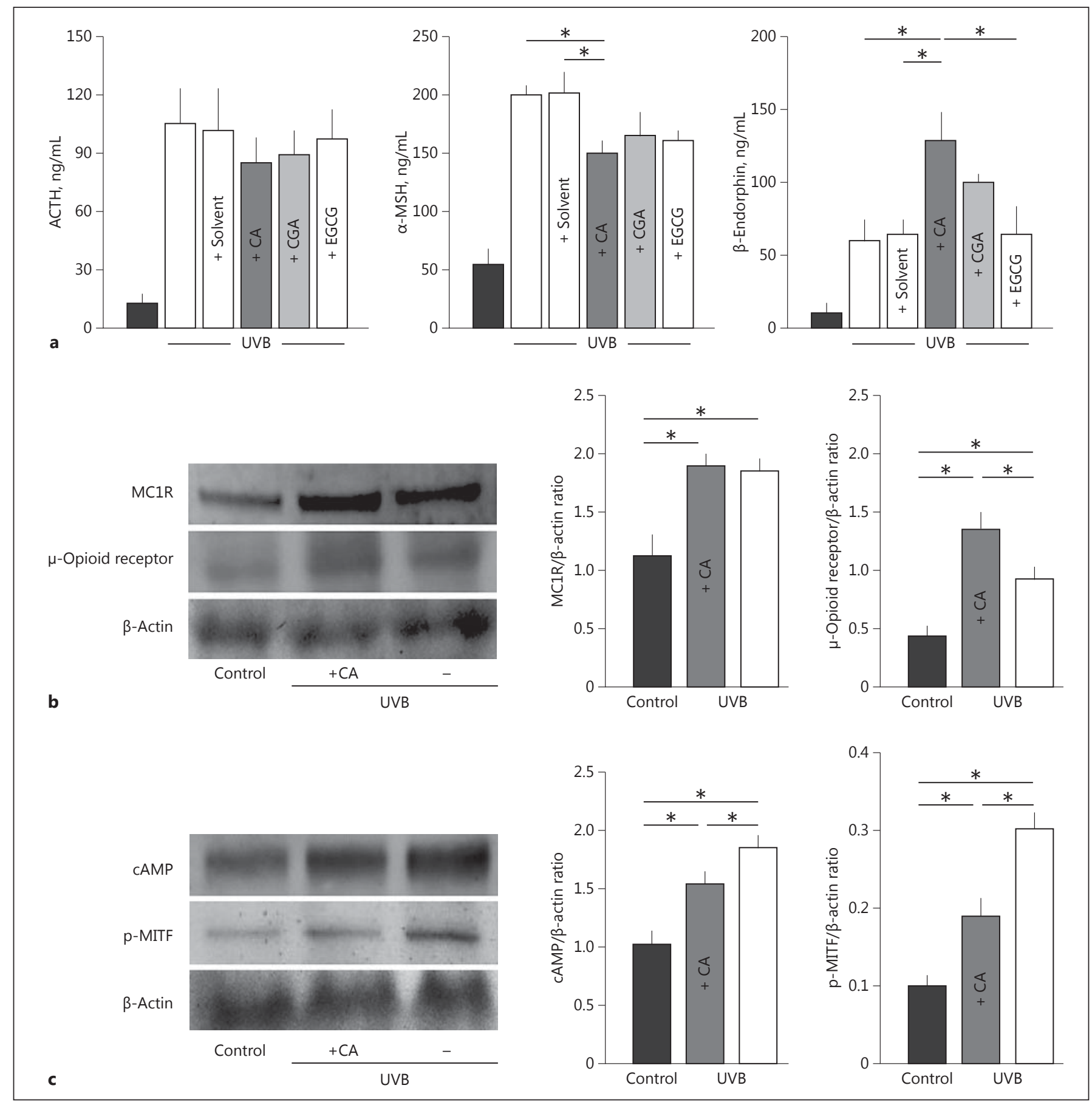

Fig. 5. The biological analysis of the mechanism underlying the ear epidermal pigmentation after UVB irradiation. a The plasma levels of $\mathrm{ACTH}, \alpha-\mathrm{MSH}$, and $\beta$-endorphin 1 day after UVB irradiation were determined by ELISA. The results were shown as the mean \pm SD derived from 6 animals. A statistical analysis was performed using an ANOVA followed by the Tukey post hoc test using SPSS (version 20) software (biological replicates; ${ }^{*} p<0.05$ ). b The expression levels of the MC1R and $\mu$-opioid receptor were determined by Western blot analysis of the ear epidermal skin samples. Equal protein loading was assessed using mouse $\beta$-actin. The results were shown as the mean \pm SD derived from 6 animals per group. A statistical analysis was performed using an ANOVA followed by the Tukey post hoc test using the SPSS (version 20) software program (biological replicates; $\left.{ }^{*} p<0.05\right)$. c The expression levels of cAMP and p-MITF were determined by Western blot analysis in the ear epidermal skin samples. Equal protein loading was assessed using mouse $\beta$-actin. The results were shown as the mean \pm SD derived from 6 animals per group. A statistical analysis was performed using an ANOVA followed by the Tukey post hoc test using the SPSS (version 20) software program (biological replicates; $*<<0.05$ ). 
Although a number of mechanisms may be involved in the up- and downregulation of skin inflammation, members of the MAPK family, including ERK1/2, c-Jun $\mathrm{N}$-terminal kinase, and p38 kinases to CREB activity, are known to be involved in common signaling pathways mediating skin inflammation after UVB irradiation [21-23]. If these proteins are not involved, then the phosphoinositol 3 (PI3)-AKT pathway is an alternative major pathway mediating CREB activity and skin inflammation after UVB irradiation [23]. CREB is a downstream target of MAPK or AKT kinase. Then we investigated which pathway was responsible for the inhibition of dermatitis by orally administered CA. We analyzed the phosphorylation of MAPK, ERK, p38, AKT, and CREB in dorsal skin samples by Western blotting (Fig. 4a, b). Our findings for Western blotting revealed that no signals through the p-p38 or p-AKT systems participated in the inhibition of the inflammatory response by CA. In the UVB irradiation + CA group, the expression of p-MAPK, p-ERK and pCREB were significantly suppressed in comparison to the UVB irradiation group.

\section{The Mechanism Underlying the Preventive Effect of}

CA against Skin Melanogenesis after UVB

One day after the final UVB irradiation session, the concentrations of $\mathrm{ACTH}, \alpha-\mathrm{MSH}$, and $\beta$-endorphin in the plasma were markedly increased by UVB irradiation exposure in mice (Fig. 5a). The level of $\alpha-\mathrm{MSH}$ in the UVB irradiation + CA group was decreased in comparison to the UVB-only and UVB + solvent groups. However, the level of $\beta$-endorphin in the UVB irradiation + CA group was significantly increased in comparison to the UVB-only and UVB + solvent and UVB + EGCG groups. With respect to the concentration of $\mathrm{ACTH}$, little difference was noted among all UVB-irradiated groups. These hormones are all produced from the proopiomelanocortin precursor protein by prohormone convertase. In order to analyze the proteins related to pigmentation, ear epidermal sheets were prepared from each group of mice 3 days after UVB irradiation. The expression of the MC1R, $\mu$-opioid receptor, cAMP, and microphthalmiaassociated transcription factor (MITF), all of which are related to skin pigmentation, in the ear epidermis for each group was examined by Western blotting (Fig. 5b, c). The MC1R, a member of the G-protein-coupled receptor family, is expressed by all cutaneous cell types and is responsive to $\alpha-\mathrm{MSH}$. The MC1R regulates the amount and type of melanin production and the number of melanocytes after UVB irradiation. In this study, the expression of the MC1R in ear skin was markedly increased by UVB irradiation, but levels were unchanged in the group administered CA (Fig. 5b). These data showed that the administration of CA inhibited pigmentation by a different pathway from the $a-M S H-M C 1 R$ pathway. Thus, we next investigated the $\beta$-endorphin- $\mu$-opioid pathway.

Mice exposed to chronic UV light displayed elevated plasma $\beta$-endorphin levels and an increase in opioid receptor signaling. $\beta$-Endorphin strongly binds to the $\mu$-opioid receptor on the membrane of melanocytes. In this study, the levels of $\beta$-endorphin in plasma and the expression of $\mu$-opioid receptor were both increased in the UVB-only group compared with the control group (Fig. 5a, b). However, these levels were increased even more in the UVB irradiation + CA group. In contrast, the expression of cAMP and p-MITF, which are located downstream of the MC1R and $\mu$-opioid receptors, was decreased in the UVB irradiation + CA group compared with the UVB-only group (Fig. 5c). These data suggest that the inhibitory effect of CA administration on pigmentation after UVB irradiation may be due to the $\beta$-endorphin- $\mu$-opioid receptor activation suppressing the cAMP-MITF pathway.

\section{Discussion}

In this study, the oral administration of CA exerted preventive effects against dermatitis and epidermal pigmentation after UVB irradiation in mice (Fig. 6). UVB irradiation is known to activate several signal pathways, including the MAPK pathway and the PI3K/AKT pathway, which have been implicated in UVB-induced skin inflammation and carcinogenesis [21-23]. MAPKs belonging to a family of serine/threonine protein kinases are activated by UVB radiation both in vitro and in vivo $[24,25]$. The MAPK family includes ERK1/2, c-Jun Nterminal kinase, and p38 kinase, which play important roles in cellular functions, such as proliferation, differentiation, and inflammation [26,27].

In this study, we investigated the phosphorylation of ERK1/2 and p38, as these signals are primarily responsible for skin inflammation after UVB irradiation. However, the PI3K/AKT pathway is also known to be activated by UVB radiation $[23,28]$. In UVB-exposed skin, the $\mathrm{PI} 3 \mathrm{~K} / \mathrm{AKT}$ signaling promotes the transcriptional activation of cyclooxygenase-2 via CREB phosphorylation, resulting in prostaglandin $\mathrm{E}_{2}$-mediated inflammation [2931]. CREB, or CRE-binding protein/activating transcription factor 1 , is located downstream of ERK1/2, p38, and $\mathrm{AKT}$ and binds to the E-box sequences in promoter re-
Skin Pharmacol Physiol 2017;30:24-35 DOI: $10.1159 / 000455237$
Yamate/Hiramoto/Sato 
Fig. 6. Diagrams summarizing the biochemical pathways by which CA exerts its protective effects. a The pathway by which orally administered CA inhibits skin inflammation after UVB irradiation. The skin keratinocytes of mice are shown. The thick gray indicator shows the effect point of CA. The inhibited pathway is surrounded by a dotted line. PI3K, phosphoinositol3-kinase; PIP2, phosphoinositol-2-phosphate; PIP3, phosphoinositol-3-phosphate; ROS, reactive oxygen species; MEK, MAPK/ERK kinase; CRE, cyclic AMP responsible element; COX-2, cyclooxygenase-2; iNOS; inducible NO synthase. b The pathway by which orally administered CA inhibits skin pigmentation after UVB irradiation is shown in the right illustration. The skin melanocytes of mice are shown. The normal pigmentation pathway after UVB irradiation is shown in the left illustration. The thick gray indicators and arrows show the effect points of CA. $\beta$-END, $\beta$-endorphin; R, receptor; Tyr, tyrosinase; TRP, tyrosinase-related protein.

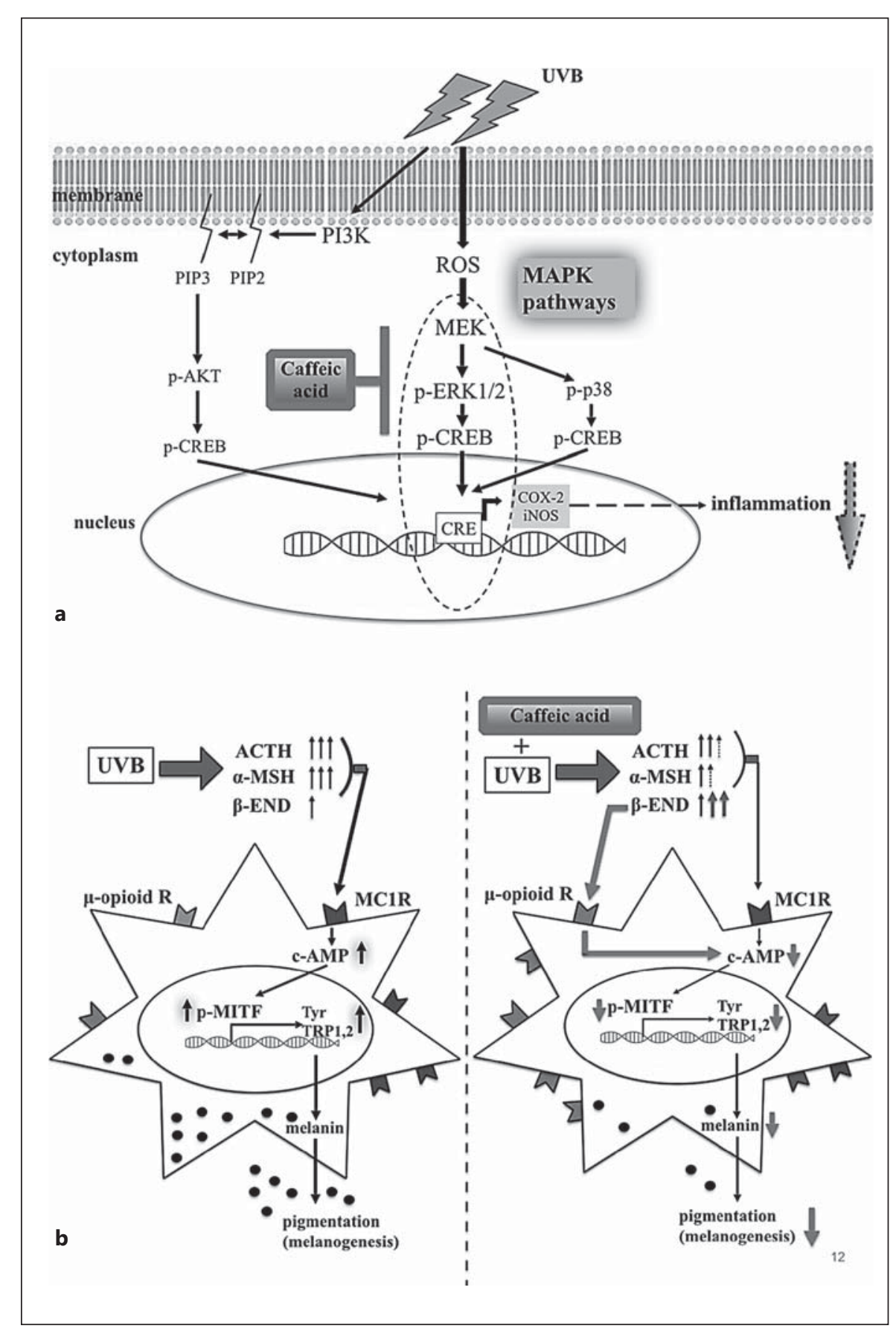

gions, triggering the transcription of numerous inflammatory genes $[32,33]$.

In this study, only the UVB irradiation + CA group showed a protective effect against dermatitis by oral administration after UVB irradiation (Fig. 2). To investigate the mechanism of this effect, we prepared dorsal skin samples 1 day after the final UVB irradiation session and then analyzed the phosphorylation of MAPK, ERK1/2, p38, AKT, and CREB. We hypothesized that the skin inflammation might be controlled at the point of the signal system where CA was located and therefore analyzed the dorsal skin after oral administration of CA using Western blotting (Fig. 4a, b). These data revealed that no signals through the p-p38 or $\mathrm{p}-\mathrm{AKT}$ systems participated in the 
inhibition of the inflammatory response by CA. In the UVB irradiation + CA group, levels of p-MAPK, pERK1/2, and p-CREB were significantly lower than in the UVB irradiation group. Taken together, these findings suggest that the suppression of skin inflammation by the oral administration of CA may proceed through the following mechanism: UVB irradiation $\rightarrow \mathrm{p}-\mathrm{MAPK} \rightarrow \mathrm{p}-$ ERK $\rightarrow$ p-CREB $\rightarrow$ inflammation.

Pigmentation is a protective response of the skin against UV-mediated damage, where melanin is produced in melanocytes in the epidermis after UV irradiation to the skin [34]. Melanin production induced by UV irradiation is regulated by several intermediary factors, such as $\alpha-\mathrm{MSH}$. $\alpha-\mathrm{MSH}$ secreted by the keratinocytes binds to the MC1R on melanocytes, leading to upregulation of cAMP, which stimulates expression of MITF [35, 36]. MITF then transcriptionally activates expression of the enzymatic machinery, including tyrosinase and tyrosinase-related protein 1 , which are critical to the synthesis of melanin within melanosomes. Chronic UV exposure has been reported to lead to an endogenous opioid-dependent state, and mice exposed to chronic UV light displayed elevated plasma $\beta$-endorphin levels and an increase in opioid receptor signaling [37].

In the present study, both the expression of the $\mu$-opioid receptor in skin and the plasma levels of $\beta$-endorphin were increased by UVB irradiation (Fig. 5). However, these changes were even further exacerbated in the UVB irradiation + CA group than in the UVB-only group, suggesting that the oral administration of CA induced an increase in the levels of $\beta$-endorphin and the expression of the $\mu$-opioid receptor. In contrast, the expression of the MC1R was not markedly changed between the UVB-only and the UVB irradiation + CA groups. Given these findings, we considered that the increase in expression of $\beta$-endorphin due to CA administration induced the subsequent expression of the $\mu$-opioid receptor, which may be involved with the decrease in the expression of cAMP.

$\beta$-Endorphin is an opioid peptide cleaved from the precursor prohormone proopiomelanocortin, from which other peptides such as ACTH, $\beta$-lipotropin hormone, and $\alpha$-MSH are also derived. $\beta$-Endorphin has been reported to exert an anti-inflammatory effect on the skin [38] and to participate in the inhibitory action of UVB-induced melanocyte activation [39]. A previous study found that $\alpha-\mathrm{MSH}$ and $\beta$-endorphin have opposing biological activities, with $\alpha-\mathrm{MSH}$ stimulating adenylate cyclase inducing an increase in cAMP while $\beta$-endorphin decreases this $\alpha-\mathrm{MSH}$-generated cAMP accumulation [40]. Given these previous findings, we considered that the inhibitory effect of pigmentation by oral administration of CA might have been exerted via a $\beta$-endorphin pathway.

However, some conflicting findings have also been reported regarding the biological activity of $\beta$-endorphin. For example, another study found that $\beta$-endorphin induces pigmentation [41], which differs from the results of the present study. We believe that this discrepancy in the findings between these studies may be due to various influences, such as differences in the degree of the increment in $\beta$-endorphin and the degree of UV irradiation applied. Further studies to clarify these issues will be needed in the future.

In the present study, in contrast to findings in the UVB irradiation + CA group, the UVB irradiation + CGA group showed no preventive effect against dermatitis and pigmentation after UVB irradiation. This lack of any positive effect may be because CGA undergoes metabolic changes deeper within the body, and so its concentration has been diluted by the time it reaches the skin surface. Future studies should investigate the difference in activity within the human body between CA and CGA after UVB irradiation.

Collectively, our results revealed that CA exerted a preventive effect against dermatitis and pigmentation after UVB irradiation in vivo. We showed that, at the concentrations evaluated in the present study, oral administration of CA exerted a remarkable inhibitory effect against skin inflammation and pigmentation (melanogenesis) after UVB irradiation. We therefore suggest that CA, which can be found in coffee, might be a good candidate compound for use in treating dermatitis and pigmentation (melanogenesis) after UV irradiation exposure.

\section{Acknowledgments}

This study was funded by the All Japan Coffee Association grant between the Suzuka University of Medical Science.

\section{Statement of Ethics}

All of the animals were treated in accordance with the animal care regulations of Suzuka University of Medical Science.

\section{Disclosure Statement}

The authors declare no conflicts of interest in association with this study.
Yamate/Hiramoto/Sato 


\section{References}

1 Kripke ML: Ultraviolet radiation and immunology: something new under the sun - presidential address. Cancer Res 1994;54:61026105.

2 Krutmann J, Morita A, Chung JH: Sun exposure: what molecular photodermatology tells us about its good and bad sides. J Invest Dermatol 2012;132:976-984.

3 Weinstock MA: Epidemiology and UV exposure. J Invest Dermatol 2013;133:E11-E12.

4 Yoshikawa T, Rae V, Bruins-Slot W, Van den Berg JW, Taylor JR, Streilein JW: Susceptibility to effects of UVB irradiation on induction of contact hypersensitivity as a risk factor for skin cancer in humans. J Invest Dermatol 1990;95:530-536.

5 Hiramoto K, Kobayashi H, Yamate Y, Ishii M, Sato EF: Intercellular pathway through hyaluronic acid in UVB-induced inflammation. Exp Dermatol 2012;21:911-914.

6 Higdon JV, Frei B: Coffee and health: a review of recent human research. Crit Rev Food Sci Nutr 2006;46:101-123.

7 Olthof MR, Hollman PC, Katan MB: Chlorogenic acid and caffeic acid are absorbed in humans. J Nutr 2011;131:66-71.

8 Zhao Z, Shin HS, Satsu H, Totsuka M, Shimizu M: 5-Caffeoylquinic acid and caffeic acid down-regulate the oxidative stress and TNF-alpha-induced secretion of interleukin-8 from Caco- 2 cells. J Agric Food Chem 2011;56:3863-3868.

9 Laure P, Michael NC, Gary W: Effect of dihydrocaffeic acid on UV irradiation of human keratinocyte HaCaT cells. Arch Biochem Biophys 2008;476:196-204.

10 Hwang SJ, Kim YW, Park Y, Lee H-J, Kim K-W: Anti-inflammatory effects of chlorogenic acid in lipopolysaccharide-stimulated RAW 264.7 cells. Inflamm Res 2014;63:81-90.

11 Yang G, Fu Y, Malakhova M, Kurinov I, Zhu F, Yao K, Li H, Chen H, Li W, Lim DY, Sheng Y, Bode AM, Dong Z, Dong Z: Caffeic acid directly targets ERK1/2 to attenuate solar UVinduced skin carcinogenesis. Cancer Prev Res 2014;7:1056-1066.

12 Ellis L, Liu W, Lim DY, Okamoto M, Qu D, Dunn JH, Fujita M: Green tea polyphenol epigallocatechin-3-gallate suppresses melanoma growth by inhibiting inflammasome and IL$1 \beta$ secretion. Biochem Biophys 2011;414: 551-556.

13 Nihal M, Roelke CT, Wood GS: Anti-melanoma effects of vorinostat in combination with polyphonic antioxidant (-)-epigallocatechin3-gallate (EGCG). Pharma Res 2010;27:11031114.

14 Hiramoto K, Yamate Y, Sugiyama D, Takahashi Y, Mafune E: Tranexamic acid suppresses ultraviolet B eye irradiation-induced melanocyte activation by decreasing the levels of pro hormone convertase 2 and alpha-melanocyte-stimulating hormone. Photodermatol Photoimmunol Photomed 2014;30:302307.
15 Yongiie M, Mingming G, Dexi L: Chlorogenic acid improves high fat diet-induced hepatic steatosis and insulin resistance in mice. Pharm Res 2015;32:1200-1209.

16 Hossen AM, Inoue T, Shinmei Y, Minami K, Fujii Y, Kamei C: Caffeic acid inhibits compound 48/80-induced allergic symptoms in mice. Biol Pharm Bull 2006;29:64-66.

17 Yoon SP, Maeng YH, Hong R, Lee BR, Kim CG, Kim HL, Chung JH, Shin BC: Protective effects of epigallocatechin gallate (EGCG) on streptozotocin-induced diabetic nephropathy in mice. Acta Histochem 2014;116:12101215 .

18 Draize JH, Woodard G, Calvery HO: Methods for the study of irritation and toxicity of substances applied topically to the skin and mucous membranes. J Pharmacol Exp Ther 1944;82:377-390.

19 Draize JH: Dermal Toxicity: Appraisal of the Safety of Chemicals in Foods, Drugs and Cosmetics. Topeka, Staff of the Division of Pharmacology Food and Drug Administration, Food and Drug Administration Officials of US Businese Office, 1959, vol 46.

20 Jimbow K, Uesugi T: New melanogenesis and photo biological processes inactivation and proliferation of precursor melanocytes from Langerhans cells. J Invest Dermatol 1982;78: 108-115.

21 Chang L, Karin M: Mammalian MAP kinase signaling cascades. Nature 2001;410:37-40.

22 Gu M, Dhanalakshmi S, Singh RP, Agarwal R: Dietary feeding of silibinin prevents early biomarkers of UVB radiation-induced carcinogenesis in SKH-1 hairless mouse epidermis. Cancer Epidemiol Biomarkers Prev 2005; 14: 1344-1349.

23 Harish CP, Mohammad A, Craig AE, Farrukh A: Fisetin inhibits UVB-induced cutaneous inflammation and activation of PI3K/AKT/ $\mathrm{NFk} \beta$ signaling pathways in SKH-1 hairless mice. Photochem Photobiol 2015;91:225234.

24 Bode AM, Dong Z: Mitogen-activated protein kinase activation in UV-induced signal transduction. Sci STKE 2003;2003:RE2.

25 Raman M, Chen W, Cobb MH: Differential regulation and properties of MAPKs. Oncogene 2007;26:3100-3012.

26 Chen W, Tang Q, Gonzales MS, Bowden GT: Role of p38 MAP kinases and ERK in mediating ultraviolet-B induced cyclooxygenase-2 gene expression in human keratinocytes. Oncogene 2001;20:3921-3926.

27 Derijard B, Hibi M, Wu IH, Barret T, Su B, Deng T, Karin M, Davis RJ: JNK1: a protein kinase stimulated by UV light and Ha-Ras that binds and phosphorylates the c-jun activation domain. Cell 1994;76:1025-1037.

28 Wan YS, Wang ZQ, Shao Y, Voorhees JJ, Fisher GJ: Ultraviolet irradiation activates PI3-kinase/AKT survival pathway via EGF receptors in human skin in vivo. Int J Oncol 2001;18:461-466.
29 Tang Q, Gonzales M, Inoue H, Bowden GT: Roles of Akt and glycogen synthase kinase 3beta in the ultraviolet B induction of cyclooxygenase- 2 transcription in human keratinocytes. Cancer Res 2001;61:4329-4332.

30 Grewe M, Trefzer U, Ballhorn A, Gyufko K, Henninger H, Krutmann J: Analysis of the mechanism of ultraviolet (UV) B radiationinduced prostaglandin $\mathrm{E}_{2}$ synthesis by human epidermoid carcinoma cells. J Invest Dermatol 1993;101:528-531.

31 Kabashima K, Nagamchi M, Honda T, Nishigori C, Miyachi Y, Tokura Y, Narumiya $S$ : Prostaglandin $E_{2}$ is required for ultraviolet $\mathrm{B}$-induced skin inflammation via EP2 and EP4 receptors. Lab Invest 2007;87:49-55.

32 Tran TT, Schulman J, Fisher DE: UV and pigmentation: molecular mechanisms and social controversies. Pigment Cell Melanoma Res 2008;21:509-516.

33 Hearing VJ: Determination of melanin synthetic pathways. J Invest Dermatol 2011; 131:E8-E11.

34 Pawelek JM, Chakraborty AK, Osber MP: Molecular cascades in UV-induced melanogenesis: a central role for melanotropins? Pigment Cell Res 1992;5:348-356.

35 Schiller M, Brzoska T, Bohm M, Metze D, Scholzen TE, Rougier A, Luger TA: Solarstimulated ultraviolet radiation-induced up regulation of the melanocortin-1 receptor, proopiomelanocortin, and alpha-melanocyte-stimulating hormone in human epidermis in vivo. J Invest Dermatol 2004;122:468476

36 Liu JJ, Fisher DE: Lighting a path to pigmentation: mechanisms of MITF induction by UV. Pigment Cell Melanoma Res 2010;23:741745 .

37 Fell GL, Robinson KC, Mao J, Woolf CJ, Fisher DE: Skin beta-endorphin mediates addiction to UV light. Cell 2014;157:1527-1534.

38 Bigliardi PL, Sumanovski LT, Buchner S, Rufli T, Rufli T, Bigliardi-Qi M: Different expression of $\mu$-opioid receptor in chronic and acute wounds and the effect of beta-endorphin on transforming growth factor beta type II receptor and cytokeratin 16 expression. J Invest Dermatol 2003;120:145-152.

39 Hiramoto K, Yamate Y, Sugiyama D, Takahashi Y, Mafune E: The gender differences in the inhibitory action of UVB-induced melanocyte activation by the administration of tranexamic acid. Photodermatol Photoimmunol Photomed 2015;32:136-145.

40 Rene F, Muller A, Jover E, Kieffer B, Koch B, Loeffler JP: Melanocortin receptors and $\delta$-opioid receptor mediate opposite signaling actions of POMC-derived peptides in CATH.a cells. Eur J Neurosci 1998;10:18851894.

41 Kauser S, Schallreuter KU, Thody AJ, Gummer C, Tobin DJ: Regulation of human epidermal melanocyte biology by beta-endorphin. J Invest Dermatol 2004;120:1073-1080. 